\title{
SPECIAL SECTION: PREVENTION
}

\section{Risk and protection factors for different intensities of adolescent substance use: when does the Prevention Paradox apply?}

\author{
TIM STOCKWELL ${ }^{1}$, JOHN W. TOUMBOUROU ${ }^{2}$, PRIMROSE LETCHER ${ }^{3}$, DIANA SMART ${ }^{4}$ \\ ANN SANSON ${ }^{34} \&$ LYNDAL BOND ${ }^{2}$
}

${ }^{1}$ National Drug Research Institute, Curtin University, ${ }^{2}$ Centre for Adolescent Health, Murdoch Children's Research Institute and Department of Paediatrics, University of Melbourne, ${ }^{3}$ Department of Psychology, University of Melbourne and ${ }^{4}$ Australian Institute of Family Studies, Australia

\begin{abstract}
The 'Prevention Paradox' applies when low-risk individuals in a population contribute the most cases of a condition or problem behaviour by virtue of their being in the majority, thereby recommending a universal or whole of population approach to prevention. The applicability of a universal as opposed to a targeted high-risk approach to the prevention of youth substance use was examined in two studies of children and adolescents conducted in Victoria, Australia. These studies were reanalysed by recombining developmental, social and individual measures to form cumulative risk indices for substance use. In Study 1 , a crosssectional survey of students, most regular tobacco, alcohol and cannabis use by 15/16-year-olds occurred in the moderate and lowrisk groups, recommending a universal prevention strategy. However, the majority of illicit drug use occurred in the highest-risk group (top 15\%). Furthermore, in younger age groups both legal and illegal drug use was concentrated mainly in the highest risk group. Study 2 used data from a major longitudinal study where risk factors at around age 11/12 years were used to predict substance use at age 17/18 years. Most students who admitted involvement in frequent smoking, heavy drinking and, although to a lesser degree, cannabis were classified as low or average risk. It is concluded that universal prevention strategies are needed for late adolescent alcohol, tobacco and cannabis use and more targeted strategies for addressing harm related to early age drug use, frequent cannabis use and illegal drug use. [Stockwell T, Toumbouru JW, Letcher P, Sanson A, Bond L. Risk and protection factors for different intensities of adolescent substance use: when does the prevention paradox apply? Drug Alcohol Rev 2004;23:67-77]
\end{abstract}

Key words: adolescents, alcohol, cannabis, epidemiology, illicit drugs, longitudinal research, risk factors, substance use, tobacco.

\section{Introduction}

The fields of public health, mental health and crime prevention have recently found common cause with growing evidence of shared developmental and social risk and protective factors influencing long-term adjustment outcomes. These factors have been identified through longitudinal and intervention research at various stages of infancy, childhood and adolescence $[1-4]$. There has also been considerable interest in the idea of there being social or structural determinants of substance use problems. For example, recent reports [5] have examined the influence of social and economic disadvantage on smoking, drinking and other drug use. Together, these two significant trends have encouraged policy makers and some practitioners to focus more on what are seen as fundamental underlying social and developmental influences rather than specific patterns of substance use per se or strategies designed to target these directly.

Tim Stockwell, National Drug Research Institute, Curtin University. John W. Toumbourou, Centre for Adolescent Health, Murdoch Children's Research Institute and Department of Paediatrics, University of Melbourne. Primrose Letcher, Department of Psychology, University of Melbourne. Diana Smart, Australian Institute of Families. Ann Sanson, Department of Psychology, University of Melbourne and Australian Institute of Families. Lyndal Bond, Centre for Adolescent Health, Murdoch Children's Research Institute and Department of Paediatrics, University of Melbourne. Correspondence to Tim Stockwell, National Drug Research Institute, Curtin University, Perth, WA 6085, Australia. E-mail: t.stockwell@curtin.edu.au

Received 1 August 2003; accepted for publication 31 October 2003.

ISSN 0959-5236 print/ISSN 1465-3362 online/04/010067-11 (C) Australian Professional Society on Alcohol and Other Drugs DOI: $10.1080 / 09595230410001645565$ 
In this paper, we sought to examine to what extent broad social and developmental risk factors in children and adolescents can explain risky substance use. To do this we conducted secondary analyses of two major studies of Australian children and adolescents. In particular, we wished to examine whether or not young people classified as high risk in these terms comprised the majority of those who engage in potentially harmful patterns of use of different drugs. Drug use intensity was considered by distinguishing recent, heavy and dependent drug use for different drug types. The analyses were considered relevant to the balance to be struck between prevention policies that address broad social and developmental risk factors and those addressing specific patterns and contexts of drug use.

If it is the case that low and/or average-risk adolescents make up the majority then, arguably, this is an illustration of the Prevention Paradox, a phrase coined by the epidemiologist Geoffrey Rose [6] in reference to how it is often the lower-risk individuals who, collectively, contribute the bulk of preventable illness in the community due to their greater numbers. Kreitman [7] first drew attention to a similar phenomenon in relation to alcohol: people who on average consume moderate amounts collectively experience more harmful consequences from drinking than do those classified as 'heavy' drinkers. In both instances it was pointed out that universal or the whole of population approaches to prevention were thus recommended, despite offering only modest benefit to most individuals - the essence of the 'paradox'. (It has been demonstrated subsequently that for population alcohol consumption, the basis for the paradox vanishes when a measure of 'binge drinking' is used instead of one of average daily consumption [7-9].

In the present paper the application of the Prevention Paradox is examined for the distribution of known social and developmental risk factors of multiple problem behaviours identifiable in Australian teenagers and their association with different patterns of alcohol, tobacco, cannabis and other illicit drug use. The analyses presented in this paper have relevance to the targeting of prevention. Where the Prevention Paradox applies, prevention investment targeted to high-risk groups are less likely to reduce population levels of drug-related harm than are those addressing the whole population regardless of risk level.

Two large Australian studies with data on social and developmental risk factors as well as intensity of drug use were reanalysed for the present study. The first of these was a cross-sectional survey of over 9000 Victorian high school children [2] which included a sample of year 11 students (average age 16.2 years). Quantity and frequency of use of alcohol, tobacco, cannabis and also other illicit drugs were assessed through retrospective self-reports. Analysis of this study enabled an examination of patterns of contemporary associations between broad risk and protective factors and intensity of drug use. The analysis was based on a research instrument developed to measure risk and protective factors in a US youth population $[10,11]$ that had been adapted successfully for use in the Australian context [2]. The instrument measured 25 risk factors and 10 protective factors that had been identified as longitudinal predictors in previous research. These factors were organized according to their influence in different developmental settings including communities, families, schools and peer groups and within individuals, as suggested through an earlier literature review [12].

The second study, the Australian Temperament Project (ATP), is a large and continuing cohort study which has followed Victorian children from infancy to 21 years of age at approximately 18month intervals using mail surveys [1,13]. A Risk Index was created comprising factors drawn from several personal and social domains assessed at ages 11-12 years that have previously been found predictive of a range of adolescent problem behaviours, including substance use. A similar classification for low-, average- and high-risk children at age 11 and 12 years (with some information collected at age $13 / 14$ years) was used in order to examine the representation of these groups among those using drugs at ages 17 and 18 years.

A novel feature of the present analysis was that different degrees and intensities of drug use were examined. Much previous research on adolescent substance use has focused on identifying which children simply use particular substances as opposed to whether their pattern of use is at a high level and whether harm or dependence are also experienced.

Simple descriptive analyses were conducted which sought to assess the extent to which high-risk children and adolescents were represented among groups who used drugs with different degrees of intensity. The Prevention Paradox was said to apply where the majority of a given group of drug users was comprised of low- and/or average-risk adolescents. In addition, the overall significance of the relationship between degree of risk and extent of drug use was examined. Based on the few available previous analyses [1,4], it was predicted that the Prevention Paradox would apply to the use of both alcohol and tobacco regardless of the intensity of that use, but would be less likely to apply to use of illicit drugs especially at higher levels of intensity. In addition, it was predicted that the cross-sectional analyses would be more likely to support the Prevention Paradox for all drug types and intensities of use. This is because the longitudinal design is a more stringent test 
of the hypothesis that the social and developmental risk factors predicted substance use.

\section{Methods}

Cross sectional analysis: Victorian Adolescent Health and Wellbeing study

The original study was conducted in 1999 on a representative sample of 9000 Victorian high school students in years 7, 9 and 11 drawn from 194 participating schools [2]. Of the students approached for parental and individual consent, $70 \%$ participated in the survey. Data from the year 11 students ( $n$ between 2439 and 2510 depending on the item) form the focus in the present analyses. Students were asked how frequently they had used different drugs in their lifetime and in the past month. In relation to alcohol they were also asked how frequently they had consumed five or more drinks on one occasion over the previous fortnight. Responses to questions about past use of cannabis, tobacco and other illicit drugs were analysed along with frequency of drinking five or more drinks on one occasion. For each drug type, two levels of drug using frequency were examined (i) use in the last month or more frequently and (ii) use in the last week or more frequently.

The assessed risk factors ranged from individual characteristics, to family, peer, school and community factors (described below). It was found that the cumulative number of elevated developmental risk factors and depressed protective factors influencing youth drug use also predicted other youth problems, including delinquency, homelessness, mental health problems and sexual risk-taking [2]. Social and developmental risk and protective factor subscales were summed across this measure to provide a single cumulative risk scale.

\section{Development of Risk Index}

All scales were based on student self-report. Response options varied and hence were adjusted to provide an average response on a four-point scale (e.g. strongly disagree, disagree, agree, strongly agree). The 25 risk factors were then summed together with the 10 reversecoded protective factor scales. Overall cut-offs for students within each school year-level utilized this aggregate risk scale and were based on differences of one standard deviation from the mean.

Community factors comprised the following:

- Low community attachment. e.g. 'I'd like to get out of my neighbourhood.'

- Community disorganization: neighbourhood perceived as physically deteriorated, with high rates of crime and drug use. e.g. 'There are fights in my neighbourhood.'

- Personal transitions and mobility: changing houses and schools, e.g. 'Have you moved house in the past year (last 12 months)?'

- Community transitions and mobility: perception that the community is not stable and that it is not easy to establish lasting personal relationships.

- Community laws/norms favourable to substance use: laws regulating alcohol and other drug use are poorly enforced. Adults believe it is 'not wrong' for minors to use alcohol or other drugs.

- Perceived availability of drugs.

- Community opportunities for prosocial involvement (protective factor, reverse coded): e.g. 'Which of the following activities for people your age are available in your community-sports teams, scouts/guides, youth groups, community service?'

- Community rewards for prosocial involvement (protective factor): e.g. 'My neighbours notice when I am doing something well and let me know.'

School factors comprised the following:

- Perceived school failure: e.g. 'Putting them altogether, what were your marks like last year?'

- Low commitment to school: e.g. 'Now, thinking back over the past year in school, how often did you try to do your best work in school?'

- School opportunities for prosocial involvement (protective factor): e.g. 'In my school, students have lots of chances to help decide things like class activities and rules.'

- School rewards for prosocial involvement (protective factor): e.g. 'My teachers praise me when I work hard in school.'

Family factors comprised the following:

- Poor family management: family rules are not clear and children are not monitored.

- Poor family discipline: e.g. 'If you wagged school would you be caught by your parents?'

- Family conflict.

- Family history of anti-social behaviour: family members have had problems with drugs and crime.

- Parental attitudes favourable to drug use: e.g. 'How wrong do your parents feel it would be for you to smoke cigarettes?'

- Parental attitudes favourable to anti-social behaviour: e.g. 'How wrong do your parents feel it would be for you to pick a fight with someone?'

- Family attachment (protective factor).

- Family opportunities for prosocial involvement (protective factor): perceived opportunities to 
participate meaningfully in the responsibilities and activities of the family.

- Family rewards for prosocial involvement (protective factor): family members praise, encourage and recognize things done well by their child.

Peer and individual factors comprised the following:

- Rebelliousness, e.g. 'I ignore rules that get in my way.'

- Early initiation of aggressive and anti-social behaviour. e.g. 'How old were you when you first attacked someone with the idea of seriously hurting them?'

- Anti-social behaviour, e.g. 'In the past year (12 months) have you carried a weapon?

- Favourable attitudes to anti-social behaviour.

- Favourable attitudes to drug use.

- Perceived risks of drug use.

- Interaction with anti-social peers, e.g. 'In the past year (12 months), have any of your best friends sold illegal drugs?'

- Friends' use of drugs.

- Sensation seeking.

- Rewards for anti-social involvement/drug use: e.g. 'What are the chances you would be seen as cool if you smoked marijuana?'

- Social skills (protective factor).

- Belief in the moral order (protective factor), e.g. 'It is important to be honest with your parents, even if they become upset or you get punished.'

- Religious involvement.

The scores of all of the year 11 students on the cumulative Risk and Protection Scale were categorized into three levels of risk:

- low risk: a score of less than 1 standard deviation below the mean (about 15\%);

- average risk: the score within one standard deviation of the mean (about 70\%); and

- high risk: a score of more than 1 standard deviation above the mean (about 15\%).

Following the definition adopted by Bond and colleagues [2], exposure to a risk factor was defined as falling into the top third of the distribution for that factor, or the bottom third for protective factors. Using this definition the average for the 35 possible elevated risk factors and depressed protective factors was 4.1 for the low-risk, 13.3 for the average-risk and 23.9 for the highrisk year 11 students.

Cross-tabulations were prepared for the level of risk and intensity of drug use for each drug type and each of the above two levels of drug use frequency. Following previous definitions [7], the Prevention Paradox was taken to apply wherever most cases of interest were comprised of low- and/or average-risk students.

\section{Measurement of substance use}

The self-completed questionnaire also covered use of tobacco, alcohol, cannabis and other illicit drug use. Items examined frequency of lifetime and monthly use and in the case of alcohol also explored for the previous 2 weeks the frequency of binge drinking (five or more drinks). For tobacco, cannabis and other illicit drug use two intensities of use were analysed: (i) at least monthly use and (ii) at least weekly use: Using on average more than four times each month. The frequency of binge drinking was analysed for at least fortnightly and for at least weekly use.

\section{Longitudinal analysis: Australian Temperament Project (ATP)}

The initial sample of the ATP comprised 2443 infants then aged 4-8 months as well as their parents who were representative of the Victorian population at that time (1983). Approximately twothirds of the sample are participating in the study after 20 years. Of the families who are no longer participating, a higher proportion are from a lower SES background, or include parents who were not born in Australia. However, there are no significant differences between the retained and no-longerparticipating subsamples on child characteristics assessed in infancy, and the retained sample resembles closely the original sample on all facets of infant functioning. Further details can be found in previous publications [14]. Thus, sample attrition is unlikely to be a significant influence when considering the impact of individual child characteristics, but may have influenced findings regarding the role of family environmental factors.

Data from the 8th, 9th and 11th waves collected in 1994, 1996 and 2000, respectively, were used for the present analysis when the participants were aged (i) 11 or 12 years, (ii) 13 or 14 years and (iii) 17 or 18 years. Measures of individual, family, peer and school risk at age $11 / 12$ (and ages $13 / 14$ in relation to family/ parents - child relationships) were used to examine the extent to which they predicted substance use at age 17/ 18. Of the 1294 children, parents and teachers with data at ages 11/12 and 13/14 years, 1064 provided complete data on substance use at age 17/18 years.

\section{Development of the Risk Index}

A total of 22 dichotomous variables were created to provide measures of developmental risk. Variables were selected based on previous research demonstrating that 
the domains they assessed were predictive of a variety of adolescent problems including substance use. All variables were assessed at 11/12 years unless specified otherwise. Variables that were assessed by more than one source of report (i.e. child self-reports, parents and teachers) were standardized and combined to form a single composite measure. Cut-offs were specified such that the most problematic $20 \%$ (or as close as possible) were designated as 'at risk' and the remaining $80 \%$ were designated as 'not at risk' (slightly different cutoffs were used for a small number of categorical or highly skewed variables, for example unemployed father). The 22 variables that were included are summarized below.

\section{Family factors}

- Parent-reported family factors/parent-child relationship: high mother's rating of child difficulty, high family stresses, father unemployed and childreported poor self-concept regarding the child's relationship with parents.

- Parent-reported parenting/parent-child relationship at 13/14 years: low parental warmth, low inductive reasoning, high use of harsh discipline, high use of physical punishment, low parental monitoring and adolescent-reported low attachment to parents.

- Family stability reported at 17/18 years: non-intact family.

School factors

- Teacher-reported school functioning: low academic competence.

Peer factors

- Parent-, teacher- and child-reported peer relationships: poor peer relationships, association with antisocial peers (parent report).

- Parent-, teacher- and child-reported social skills: low social skills.

Individual factors

- Parent-reported Temperament: low persistence, high negative reactivity, high activity

- Parent-, teacher- and child-reported behaviour problems: hostile-aggressive, hyperactive, anxiousfearful, depressed.

Further details about the particular scales used are available from Prior et al. [13]. Participants were used in the foregoing analyses only if complete data were available on at least 18 of the 22 variables.
Measurement of substance use and dependence at 17/18 years

A self-completed questionnaire covering use of tobacco, alcohol and cannabis was administered with items regarding lifetime use, quantity and frequency of drug use in the last 30 days, experience of drug-related harm and dependence. Categories of drug users meeting criteria for increasing intensity of use were defined separately for each drug as follows:

(i) Lifetime users: those who reported smoking two or more cigarettes or drinking two or more alcoholic drinks or trying cannabis ever in their lives.

(ii) Recent users: those who reported smoking two or more cigarettes or drinking five or more alcoholic drinks in quick succession or smoking at least two joints/cones of cannabis on at least one of the last 30 days.

(iii) Heavy users: those who reported smoking five or more cigarettes or drinking five or more alcoholic drinks in quick succession or smoking at least two joints/cones of cannabis on at least 4 of the last 30 days.

(iv) Dependent users: for tobacco, those who reported smoking an average of at least five cigarettes per day in the last 30 days; for alcohol, those who responded 'sometimes' or 'often' to at least one of the following questions: 'Did your use of alcohol cause you to feel you were not able to stop drinking once you started?'; 'Did your use of alcohol cause you to feel irritable or depressed when it wasn't available?'; and for cannabis, those who responded 'sometimes' or 'often' to at least one of the following questions: 'Did your marijuana use cause you to feel you couldn't stop using it?' or 'Did your marijuana use cause you to feel irritable or depressed when it wasn't available?'

The scores of all participants with complete data both on substance use and the Risk Index were categorized into three levels of risk to match as closely as possible the frequency distribution employed in the first study:

- low risk: a score of 0 on the risk index (12.8\%);

- average risk: a score of between 1 and 7 on the risk index (74.1\%);

- high risk: a score of 8 or more on the risk index $(13.2 \%)$.

Cross-tabulations were prepared for the level of risk and intensity of drug use for each drug type and each of the above four levels of drug use frequency. Again, the Prevention Paradox was taken to apply wherever most 
cases of interest were comprised of low- and/or averagerisk participants.

As a sensitivity test, some of these descriptive analyses were repeated with less stringent criteria for what constituted both a low-risk and a high-risk adolescent. The frequency distribution was divided as close as possible to thirds as follows:

- low risk: a score of 0 or 1 on the risk index $(30.0$ $\%)$

- average risk: a score of between 2 and 4 on the risk index $(38.5 \%)$;

- high risk: a score of 5 or more on the risk index $(31.5 \%)$.

\section{Results}

Cross-sectional analyses: Victorian Adolescent Health and Wellbeing study

(i) At least monthly use of different drugs. As shown in Fig. 1 , at this frequency of drug use the Prevention Paradox applies both to tobacco and 'binge' drinking (at least fortnightly), does so marginally for cannabis use and does not apply in relation to other illicit drug use. Among the relatively small number reporting use of other illicit drugs in the last 30 days (130 of 2510), $61.5 \%$ were identified as high-risk adolescents on the Risk and Protection Scale. Very few low-risk students reported use of any drugs.

(ii) At least weekly use of different drugs. As shown in Fig. 2 , while over a third of both at least weekly smokers and also of students reporting drinking five or more drinks in succession at least once a week were high risk, the Prevention Paradox still applied to users of these legal drugs at this level of intensity. The Prevention Paradox did not apply to either category of illicit drug use with $60 \%$ of cannabis users and $69 \%$ of users of other illicit drugs being categorised as high-risk adolescents. Very few low-risk students reported using any drugs, legal or otherwise.

These analyses were repeated with students in high school years 9 and 7 (graphs not shown) and with few exceptions the Prevention Paradox did not apply. The Prevention Paradox applied only to at least monthly or weekly tobacco use and to fortnightly binge drinking for year 9 students (average age 14.2). For year 7 students (average age 12.3) the Prevention Paradox did not apply - the majority of drug use occurred among the high risk subjects. For example, $58 \%$ of at least monthly tobacco use occurred in this group. For all other substances that were used monthly or more often in year 7 no less than $66 \%$ occurred in the high-risk group.

\section{Longitudinal analysis: Australian Temperament Project (ATP)}

(i) Lifetime drug users. As shown in Fig. 3, the distribution of risk status in early adolescence closely parallels rates of lifetime use of tobacco, alcohol or cannabis at $17-18$ years. The Pearson $\chi^{2}$ test, however, shows that participants reporting any lifetime cannabis use are slightly more likely to be high risk in early adolescence. For all three drug types, the Prevention Paradox applies and close to three-quarters of all late adolescent substance users

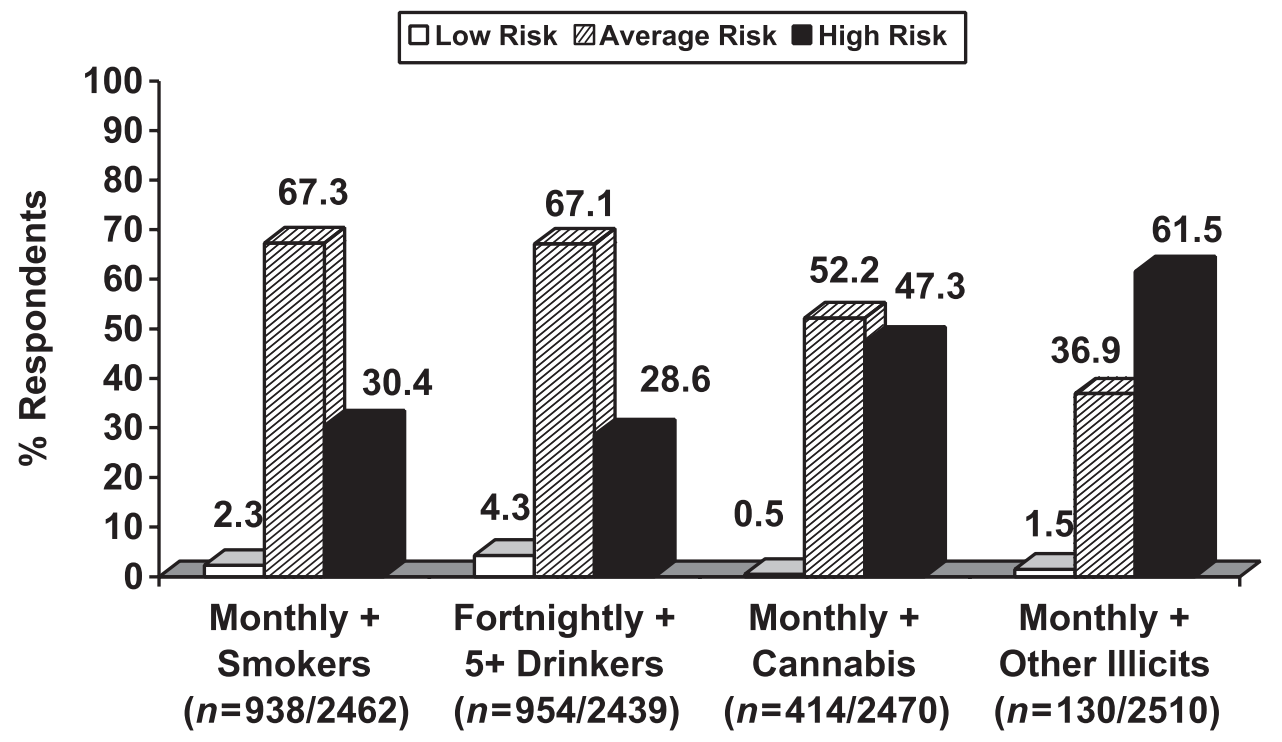

Figure 1. Broad risk status of students aged 16.2 years (mean) who use different drugs at least monthly (or fortnightly for 'binge drinking'): Health and Wellbeing Study. 


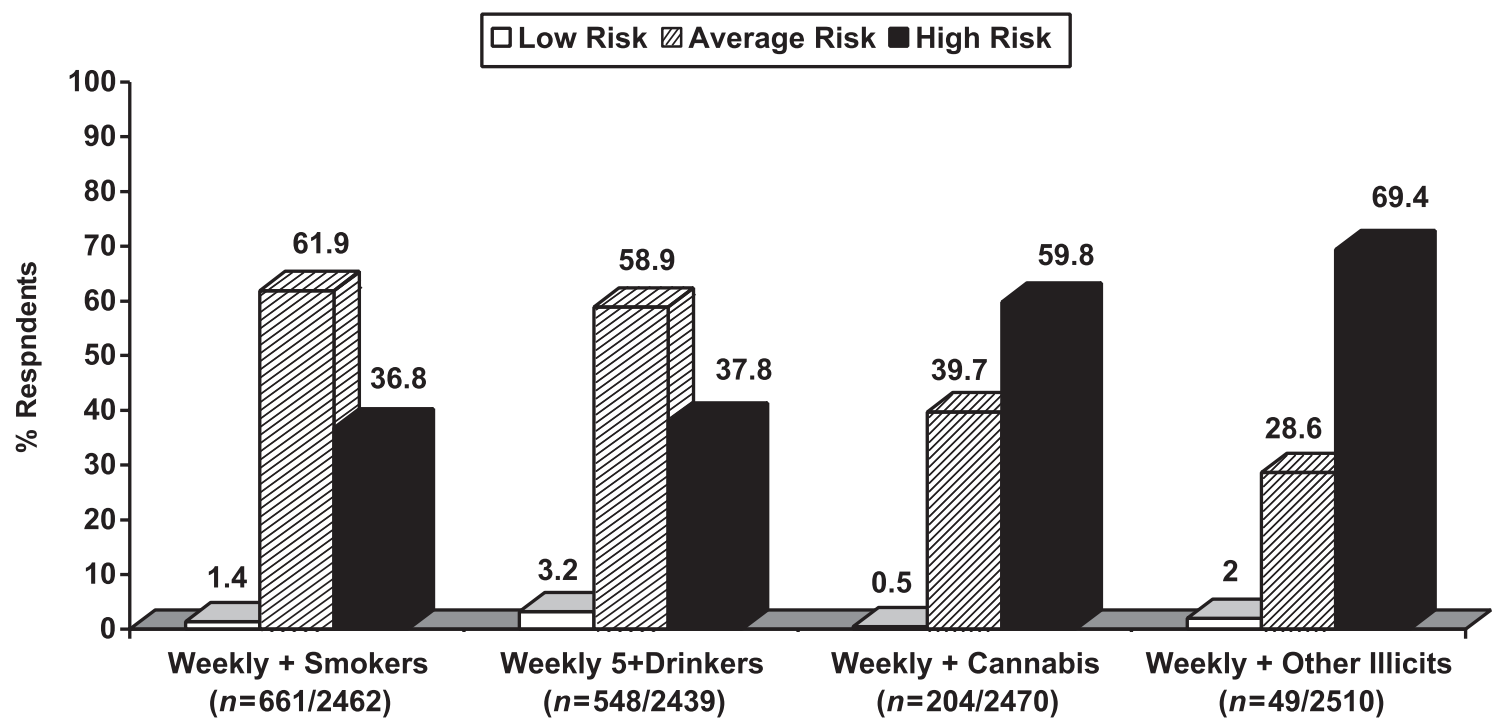

Figure 2. Broad risk status of students aged 16.2 years (mean) who use different drugs at least weekly: Health and Wellbeing Study.

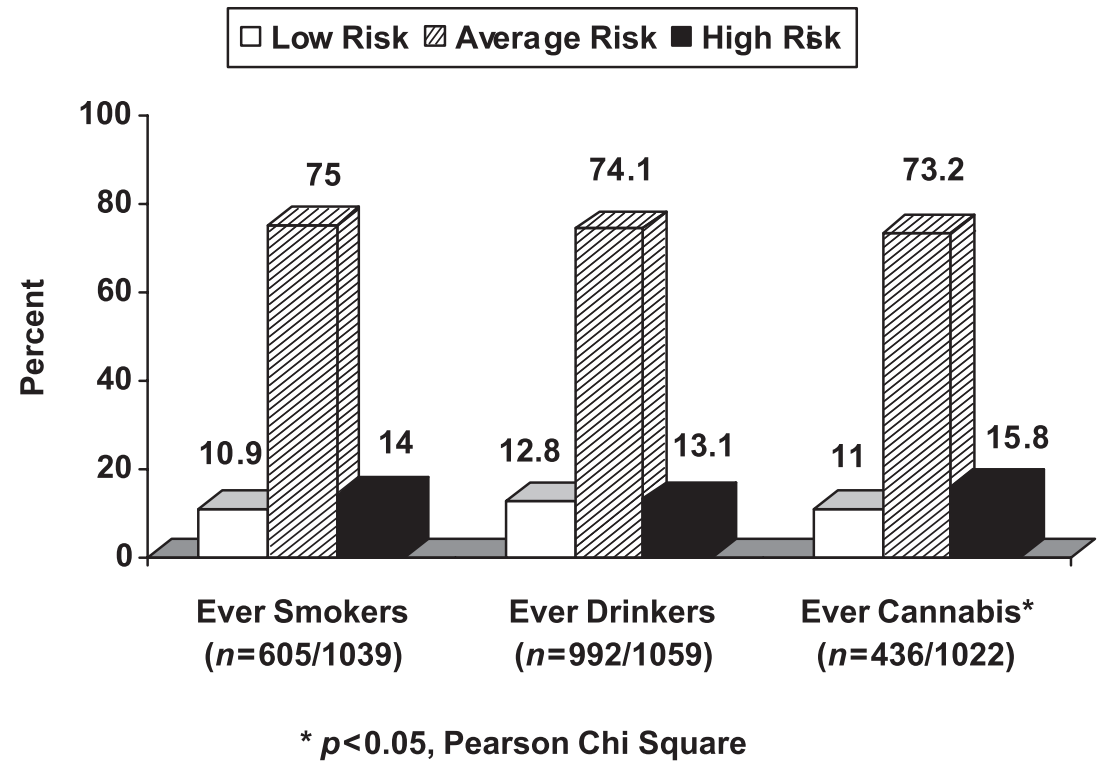

Figure 3. Broad risk status of children aged 11-12 who use different drugs by age 17-18 years.

are designated to be of average risk in early adolescence.

(ii) Last 30 day users. Fig. 4 shows that the Prevention Paradox still applies at this level of drug use intensity with close to three-quarters of all respondents being classified as average risk in early adolescence in relation to use of each drug type. There is a highly significant tendency, however, for respondents who reported cannabis use in the last 30 days to be high risk, while the rates were lower among the low-risk groups. (iii) 'Heavy' drug users. As shown in Fig. 5, those who reported more frequent and heavier drug use were significantly more likely to have been assessed as high risk in early adolescence and less likely to have been low risk. However, the Prevention Paradox still held strongly for each drug type, with the bulk of users coming from average-risk groups.

(iv) Dependent users. As shown in Fig. 6, those 17- and 18-year-olds who reported at least one dependence sign in the last 30 days were significantly more likely to have 


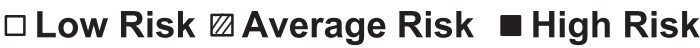

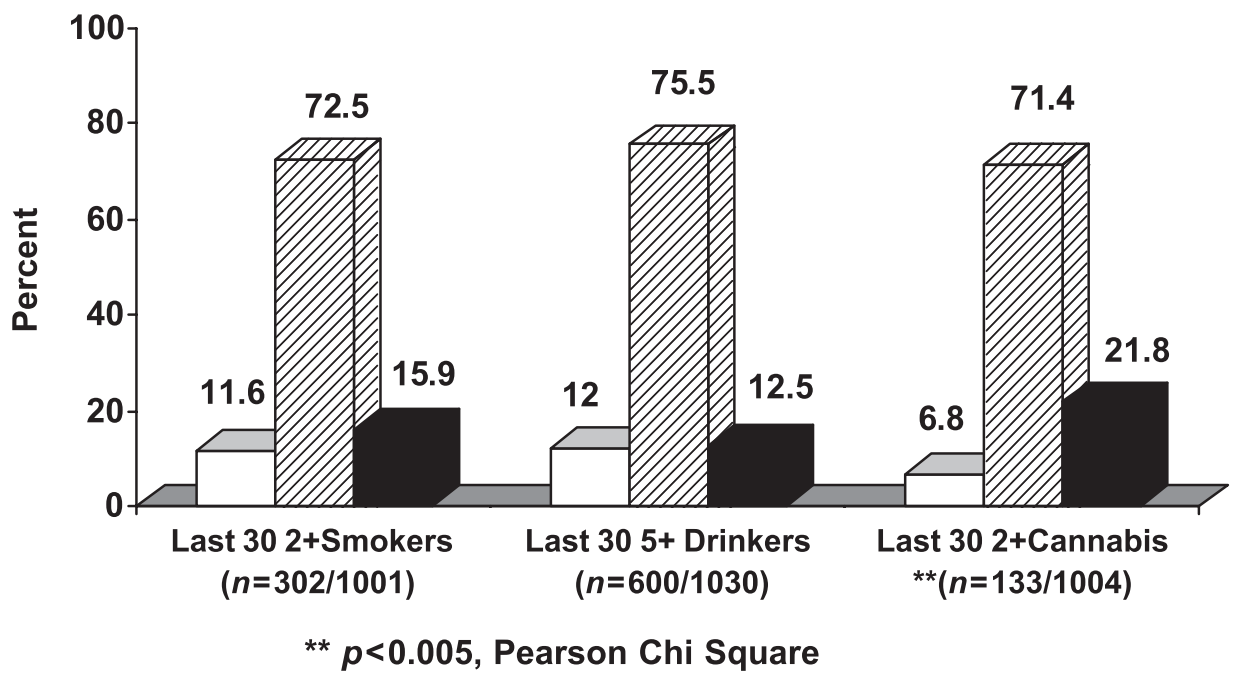

Figure 4. Broad risk status of adolescents who later used different drugs in last 30 days: ATP Study.

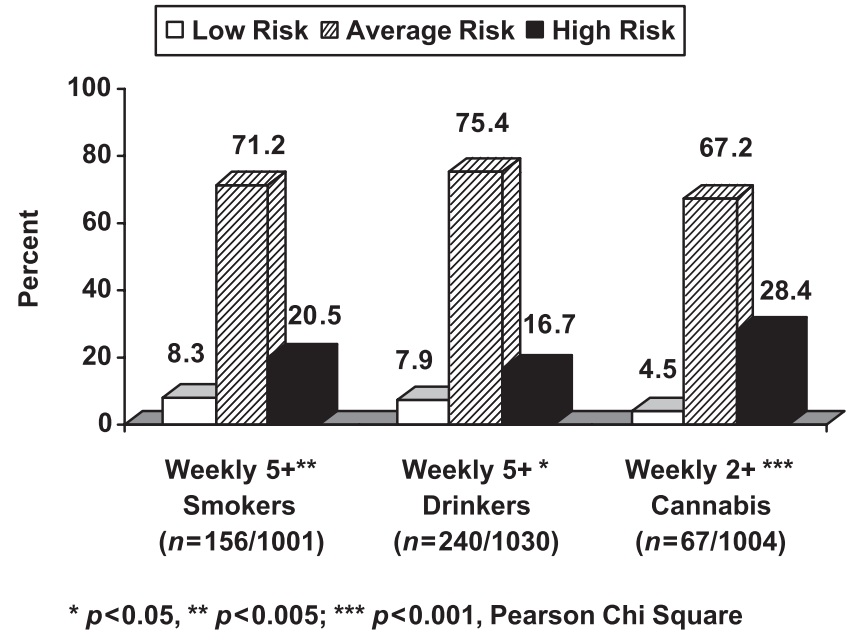

Figure 5. Broad risk status of adolescents who become frequent heavy users by age 17-18 years: ATP Study.

been assessed as high risk in early adolescence and less likely to have been low risk. Once again, however, the Prevention Paradox still applied in that the great majority of respondents so categorised were Average Risk children in early adolescence.

(v) Sensitivity test for heavy drug users. When the much less stringent criteria both for low- and high-risk adolescents was applied in which the sample was divided broadly into thirds on the basis of their Risk Index score, the Prevention Paradox still applied for the smokers and drinkers but narrowly failed to do so for

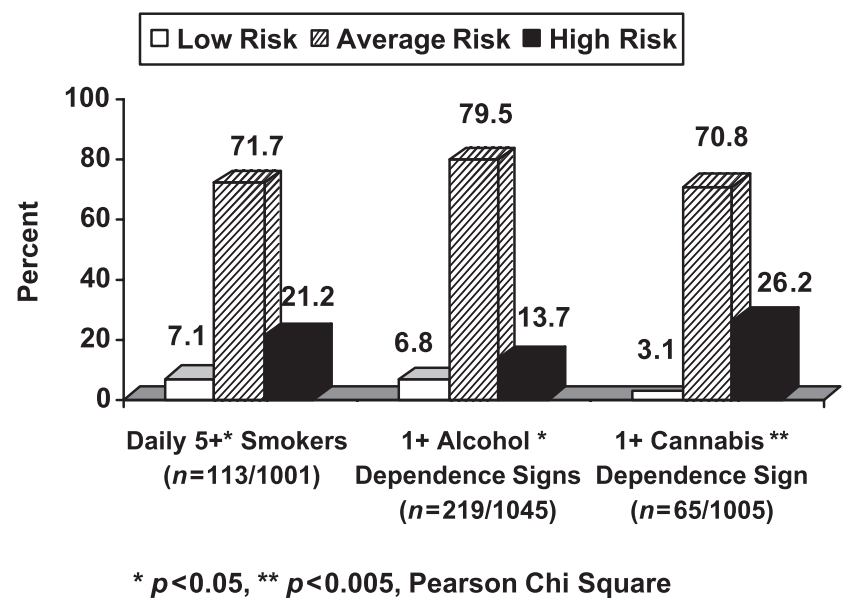

Figure 6. Broad risk status of adolescents who show signs of drug dependence by age 17-18 years: ATP Study.

the cannabis users. In each case there was a highly significant effect for drug types that users at this level of intensity were more likely to have been assessed as high risk and less likely to have been low risk as adolescents (see Fig. 7).

\section{Discussion}

As anticipated, the measures of social and developmental risk and protection predicted more hazardous drug use in both the cross-sectional and the longitudinal studies of Australian adolescents. This association was more pronounced for the illicit drugs and also 


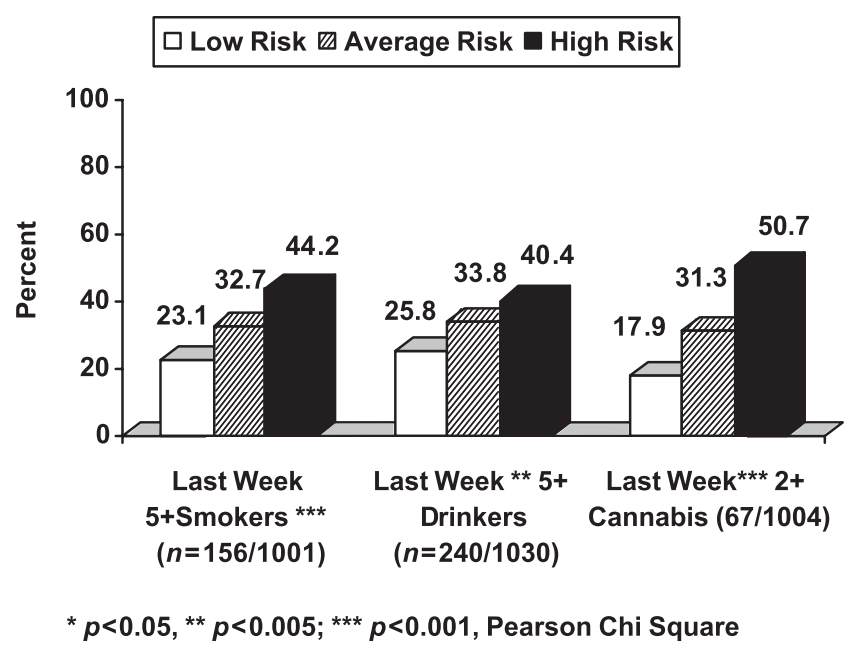

Figure 7. Broad risk status of adolescents (divided into thirds) who report frequent heavy drug use in last 30 days: ATP Study.

for more intense levels of use of each drug type. Also, as predicted, the relationship between the broad social and developmental risk status of the adolescents and their use of drugs was much closer and more significant in the cross-sectional than in the longitudinal study. There are several possible explanations for this latter finding:

1. The relatively strong associations found in the cross-sectional study may be due partly to some items measured in the adapted Health and Wellbeing Risk and Protection Scale being the outcomes of concurrent drug use rather than the cause. In this area temporal influences are likely to be reciprocal, for example arguments with parents, poor performance at school and having drug-using peers are likely to be inter-related in complex ways over time with drug use.

2. Across-time relationships are almost invariably less powerful than concurrent associations, particularly over the relatively long time span of 6 years employed by the ATP study. It is also worth noting that this time span covers a period of significant developmental change, including the transition from elementary to secondary school and the onset of puberty. Additionally, there can be considerable fluidity in developmental pathways, even as late as the adolescent years [15]. Thus, the less powerful longitudinal findings may be expected. Nevertheless, longitudinal connections were evident: problematic characteristics present in childhood were found to be significant risk factors for later adolescent substance use.

3. The two risk indices differed somewhat in content. For example, while the longitudinal ATP study had an extensive range of measures assessing individual characteristics and aspects of the family environment derived from surveys of parents, teachers and the children themselves, it did not possess the range of community and school risk factors contained in the crosssectional study. The ATP study did not include a number of early adolescent risk factors that are known to be highly predictive of later adolescent drug use including early age drug use, parent and peer drug use. Similarly, the two studies differed somewhat in the criteria developed to define substance use.

4. There was a slightly more stringent definition of what constituted high-risk adolescents in the longitudinal ATP study-13\% versus $15 \%$ of the participants in the cross-sectional Victorian school survey. The overall pattern of results remained, even when the definition of high risk was broadened in the ATP study, suggesting this small difference alone would not account for the large difference between the results of these two studies.

5. Attrition of participants in the longitudinal ATP study may have diminished some associations, as high-risk youth who were also heavy drug users may have been difficult to retain in the longitudinal study.

6. The age at which drug use was assessed was different in the two studies. The analysis of the Health and Wellbeing Study revealed that the Prevention Paradox applied to a greater extent as youth moved from early to late adolescence. Although at an early age heavy drug use may be somewhat restricted to high-risk groups as youth move toward young adulthood, those at average risk also appear to become involved in these behaviours.

This pattern of results strongly supports the view that social and developmental risk factors in late childhood and early adolescence contribute significantly to frequent and heavy drug use and dependence in the late teenage years. These risk factors are especially important in relation to later heavy use of illicit drugs. In the cross-sectional analyses the Risk and Protection scale was associated with monthly or fortnightly drug use, but ever having used drugs was not assessed in this study. The ATP risk factors were not found, however, to predict which children would subsequently report having ever used tobacco or alcohol, although cannabis use was predicted weakly.

Having confirmed and added to previous research on predictors of adolescent substance use, the main interest of the analyses presented here was an assessment of for which drugs and for which levels of intensity of use did the Prevention Paradox apply, and so a 
universal or whole-population approach to prevention is recommended. The paradox held for both alcohol and tobacco use at each level of intensity examined and in both datasets from around age 16. Thus even for regular 'binge' drinking $(5+$ drinks in quick succession or per occasion) and also for displaying one or more signs of dependence (in the ATP), most of the adolescents meeting these criteria were classified as average risk on the social and developmental risk/ protection indices assessed in childhood. This finding applied even when a much less stringent criterion was applied for high-risk status in the ATP, i.e. the top third of adolescents on this parameter.

Although there appear to be substantial numbers of average-risk youth engaging in frequent, heavy or dependent drug use there was also evidence of a protective effect for youth with low risk. Although the ATP children of low risk reported ever and monthly use of tobacco and binge alcohol use, they were less involved in heavy or dependent use. It may be that community prevention programmes that act to reduce risk factors and enhance protective factors across multiple settings (family, schools, community organization and drug availability) have more success in preventing youth drug use.

There was evidence that the Prevention Paradox diminished for early youth involvement in frequent and heavy drug use and as drug use progression moved away from ever use toward the heavy and dependent categories. It is possible that stricter indices of drugrelated harm such as deaths, hospital admissions or drug treatment entry may also show less evidence for the Prevention Paradox.

Contrasting results were obtained for the illegal drugs: in the cross-sectional Victorian school survey, the Prevention Paradox held only marginally for monthly use of cannabis at age 16 but not for weekly cannabis use or use of other illicit drugs either weekly or monthly. However, when using the more stringent test of the longitudinal ATP study, the Prevention Paradox held for any frequency of cannabis use including signs of dependence. Only when the much less strict definition of a high-risk adolescent was applied did the Prevention Paradox narrowly fail to hold, i.e. just over $50 \%$ of the 17 - and 18 year-olds who met the criteria for frequent heavy use of cannabis had been categorized as high-risk children at age 11 or 12 years.

These analyses speak to the issue of sensitivity (rates of problems among those with differing levels of risk). While outside the scope of the present paper, it would also be valuable to explore the issue of specificity (the distribution of risk among those with the problem outcome), which would clarify further the connections between risk status and adolescent substance use.

Some important conclusions for prevention policy can be drawn from these analyses. First, in these samples the great majority of teenagers who drank excessively and/or frequently smoked cigarettes had not had extremely socially, developmentally or economically deprived backgrounds. This suggests that a comprehensive prevention policy must include elements that have universal applicability to young people rather than a more selected high-risk group. For example, policies that address the issues of availability, price and marketing must not be neglected. Consideration of universal strategies that address risk and protection factors within the whole community (e.g. broad social programmes to reduce family discord) should also be considered. Furthermore, given the high prevalence of these health-compromising behaviours it is also wise to develop policies which have the potential to reduce the harmful effects of alcohol and tobacco by paying attention to drug use environments and the type of products being marketed. A second conclusion is that adverse social and developmental factors are more significant in the genesis of illicit drug use than they are for alcohol and tobacco, especially for illicit drugs other than cannabis. The longitudinal ATP study indicates, however, that the great majority of 17- and 18-year-olds who report frequent cannabis use were not classified as socially or developmentally disadvantaged or at risk in late childhood. While it is especially important to consider how to address social and developmental risk factors in the prevention of illicit drug use and other adolescent problem behaviours, it is still the case that a broader range of universal strategies need to be considered. For example, laws to deter the sale and supply of cannabis need to be designed with a view to minimizing the potential social and legal harms associated with the application of criminal sanctions [16].

These findings also have implications for the targeting of social and developmental prevention programmes in early adolescence. The ATP findings showed that healthy development at the end of childhood yielded only a small protective benefit against engagement in potentially harmful levels of drug use in adolescence. The risk index employed in the ATP did not include important risk factors for harmful drug use in early adolescence such as early age drug use and peer and parent drug use. In contrast, the cross-sectional findings demonstrated that the Prevention Paradox broke down when monthly and weekly drug use was assessed in the early years of high school. These findings suggest the potential relevance of targeted prevention programmes addressing drug use in early high school. It is possible that high levels of early age drug use among high-risk students may lead to peer influences in high schools and within the community more generally that may subsequently lead many lowand average-risk students to engage in potentially harmful drug use. 
In summary, applying the social and developmental risk and protection approach in childhood to the prevention of substance use problems holds some exciting potential but is by no means a panacea. Given the markedly greater prevalence of heavier use and harm associated with tobacco and alcohol, it is especially important to maintain an emphasis on regulatory strategies with universal application and proven effectiveness to minimise health and safety consequences associated with the misuse of these drugs. Community prevention approaches that combine an emphasis on preventing developmental risk and strengthening protective factors while also strengthening universal regulatory systems and harm-reduction may have particular promise [4]. There is, however, definite value in also pursuing the social and developmental risk and protection factor approach especially in relation to the prevention of the risky use of illicit drugs and the use of drugs in early adolescence.

\section{Acknowledgements}

Data collection for the Victorian Health and Wellbeing study was funded by the Victorian Department of Human Services, and managed by Ms Lyndal Thomas. We wish to acknowledge the role of Professors Frank Oberklaid and Margot Prior in initiating the Australian Temperament Project and the support of the National Health and Medical Council in funding the adolescent follow-up. An early version of this paper was presented at the Kettil Bruun Society Thematic Meeting 'Preventing Substance Use, Risky Use and Harm: What is Evidence-based Policy?', Fremantle, Western Australia, 24-27 February, 2003.

\section{References}

[1] Williams B, Sanson A, Toumbourou JW, Smart D. Patterns and predictors of teenagers' use of licit and illicit substances in the Australian Temperament Project cohort. Melbourne: University of Melbourne, 2000.

[2] Bond L, Thomas L, Toumbourou JW, Patton GC, Catalano R. Improving the lives of young Victorians in our community: a survey of risk and protective factors. Melbourne: Department of Human Services, 2000: 1-390.
[3] Lewinsohn PM, Roberts RE, Seeley JR, Rohde P, Gotlib IH, Hops H. Adolescent psychopathology: II. Psychosocial risk factors for depression. J Abnorm Psychol 1994;103:302-15.

[4] Loxley W, Toumbourou J, Stockwell $\mathrm{T}$ et al. The prevention of substance use, risk and harm in Australia. A review of the evidence. Melbourne: National Drug Research Institute and the Centre for Adolescent Health, 2003:1431.

[5] Spooner C, Hall W, Lynskey M. Structural determinants of youth drug use. Canberra: Australian National Council on Drugs, 2001.

[6] Rose G. Sick individuals and sick population. Int J Epidemiol 1985;14:32-8.

[7] Kreitman N. Alcohol consumption and the preventive paradox. J Addict 1986;81:353-63.

[8] Stockwell T, Hawks D, Lang E, Rydon P. Unravelling the preventive paradox for acute alcohol problems. Drug Alcohol Rev 1996;15:7-15.

[9] Gmel G, Klingemann S, Muller R, Brenner D. Revising the preventive paradox: the Swiss case. Addiction 2001;96:273-84.

[10] Arthur MW, Hawkins JD, Pollard JA, Catalano RF, Baglioni AJ. Measuring risk and protective factors for substance use, delinquency, and other adolescent problem behaviors: the Communities That Care Youth Survey. Eval Rev 2002;26:575-601.

[11] Pollard JA, Hawkins JD, Arthur MW. Risk and protection: are both necessary to understand diverse behavioral outcomes in adolescence? Social Work Res 1999;23:145-58.

[12] Hawkins JD, Catalano RF, Miller JY. Risk and protective factors for alcohol and other drug problems in adolescence and early adulthood: implications for substance abuse prevention. Psychol Bull 1992;112:64-105.

[13] Prior M, Sanson A, Smart D, Oberklaid F. Pathways from infancy to adolescence. Australian Temperament Project 1983-2000. Melbourne: Australian Institute of Family Studies, 2000.

[14] Prior M, Sanson A, Smart D, Oberklaid F. Psychological disorders and their correlates in an Australian community sample of pre-adolescent children. J Child Psychol Psychiatry 1999;40:563-80.

[15] Smart D, Vassallo S, Sanson A et al. Patterns and precursors of adolescent antisocial behaviour-second Report. Melbourne: Crime Prevention Victoria, 2003.

[16] Lenton S, Heale P, Erickson P. The regulation of cannabis possession, use and supply. A discussion document prepared for the Drugs and Crime Prevention Committee of The Parliament of Victoria. National Drug Research Institute Monograph 3. Perth: National Drug Research Institute, 2000. 